T. Adachi

Nagoya Math. J.

Vol. 104 (1986), 55-62

\title{
MARKOV FAMILIES FOR ANOSOV FLOWS WITH AN INVOLUTIVE ACTION
}

\author{
TOSHIAKI ADACHI
}

\section{$\S 1$. Introduction}

The aim of this note is to construct "involutive" Markov families for geodesic flows of negative curvature. Roughly speaking, a Markov family for a flow is a finite family of local cross-sections to the flow with fine boundary conditions. They are basic tools in the study of dynamical systems. In 1973, R. Bowen [5] constructed Markov families for Axiom $A$ flows. Using these families, he reduced the problem of counting periodic orbits of an Axiom $A$ flow to the case of hyperbolic symbolic flows.

It is well-known that geodesic flows of negative curvature are Anosov flows, hence are of Axiom $A$ type. But within the class of Anosov flows the geodesic flows still retain a special importance. Let $\psi_{t}$ be the geodesic flow on the unit tangent bundle $U N$ of a compact manifold $N$ of negative curvature. If we define an involution $\theta: U N \rightarrow U N$ by $\theta(v)=-v$, then we have $\psi_{t} \circ \theta=\theta \circ \psi_{-t}$. This is a typical property and should be utilized for getting some informations on geodesics. For applying Bowen's symbolic dynamics, Markov families which inherit this involutive property are very useful. In the forthcoming paper [2], we shall show that there exist infinitely many prime closed geodesics in each one-dimensional homology class of $N$. By using our preliminary work, one can reduce the problem to the case of "involutive" graphs.

Our construction goes on the same lines as in [5]. Since it is not difficult to fill up between the lines, we just point out where should be modified.

The author is grateful to Professors K. Shiraiwa and T. Sunada for valuable advice.

Received February 7, 1985. 


\section{§. Involutive Markov families}

A differentiable flow $\varphi_{t}$ on a compact Riemannian manifold $M$ is called Anosov if the following condition holds. The tangent bundle of $M$ can be written as the Whitney sum of three $d \varphi_{t}$-invariant continuous subbundles

$$
T M=E^{T} \oplus E^{s} \oplus E^{u},
$$

where $E^{T}$ is the line bundle tangent to the flow, and there are constants $C, \lambda>0$ such that

$$
\begin{array}{ll}
\left\|d \varphi_{t}(v)\right\| \leq C e^{-\lambda t}\|v\| \quad \text { for } \quad v \in E^{s}, \quad t \geq 0 \\
\left\|d \varphi_{-t}(v)\right\| \leq C e^{-\lambda t}\|v\| \quad \text { for } \quad v \in E^{u}, \quad t \geq 0 .
\end{array}
$$

A finite family of closed sets $\mathscr{T}=\left\{T_{1}, \cdots, T_{n}\right\}$ is called a proper family of size $\alpha$ if the followings hold;

1) $M=\bigcup_{i=1}^{n} \varphi_{[-a, 0]}\left(T_{i}\right)$,

2) there are differentiable closed disks $D_{1}, \cdots, D_{n}$ transverse to $\varphi_{t}$ of diameter smaller than $\alpha$ such that

a) $\operatorname{dim}\left(D_{i}\right)=\operatorname{dim}(M)-1$,

b) $T_{i} \subset \operatorname{Int}\left(D_{i}\right)$ and $T_{i}=\overline{\operatorname{Int}}_{D_{i}}\left(T_{i}\right)$, where $\operatorname{Int}_{D_{i}}\left(T_{i}\right)$ is the interior of $T_{i}$ with respect to the relative topology of $D_{i}$,

c) for $i \neq j$, at least one of the sets $D_{i} \cap \varphi_{[0, a]}\left(D_{j}\right)$ and $D_{j} \cap \varphi_{[0, a]}\left(D_{i}\right)$ is empty.

For each $x \in \Gamma(\mathscr{T})=\bigcup_{i=1}^{n} T_{i}$ let $0<t_{\mathscr{T}}(x) \leq \alpha$ be the least time for which $\varphi_{t}(x) \in \Gamma(\mathscr{T})$. We define a bijection $H_{\mathscr{T}}: \Gamma(\mathscr{T}) \rightarrow \Gamma(\mathscr{T})$ by $H_{\mathscr{T}}(x)=$ $\varphi_{t_{\mathscr{F}}(x)}(x)$ and a dense subset of $\Gamma(\mathscr{T})$ by

$$
\Gamma^{\prime}(\mathscr{T})=\left\{x \in \Gamma(\mathscr{T}) \mid H_{\mathscr{T}}^{k}(x) \in \bigcup_{i=1}^{n} \operatorname{Int}_{D_{i}}\left(T_{i}\right) \text { for any } k \in Z\right\} .
$$

Let $\prod_{z} \mathscr{T}$ be the set of all doubly infinite sequences of symbols with the product topology. There is a continuous injective map $Q: \Gamma^{\prime}(\mathscr{T}) \rightarrow \prod_{z} \mathscr{T}$ given by

$$
Q(x)=\left(q\left(H_{\mathscr{F}}^{k}(x)\right)\right)_{k \in Z},
$$

where $q(y)$ denotes the unique element of $\mathscr{T}$ containing $y$. Since $Q^{-1}$ : $Q\left(\Gamma^{\prime}(\mathscr{T})\right) \rightarrow \Gamma(\mathscr{T})$ is Lipschitz with respect to the canonical distance on $\prod_{z} \mathscr{T}$, one can define a surjective map $\pi: \overline{Q\left(\Gamma^{\prime}(\mathscr{T})\right)} \rightarrow \Gamma(\mathscr{T})$ as the continuous extension of $Q^{-1}$. We define a strictly positive function $f_{\mathscr{T}}$ : $\bar{Q}\left(\Gamma^{\prime}(\mathscr{T})\right) \rightarrow(0, \alpha]$ as the continuous extension of $t_{\mathscr{F}} \circ Q^{-1}$. In general, the 
set $\overline{Q\left(\Gamma^{\prime}(\mathscr{T})\right)}$ is complicated. To aboid this we have to choose $\mathscr{T}$ carefully. For a point $x \in M$ and $\varepsilon>0$ we define the stable and unstable sets by

$$
\begin{aligned}
& W_{\varepsilon}^{s}(x)=\left\{\begin{array}{ll}
y \in M & \begin{array}{l}
d\left(\varphi_{t}(x), \varphi_{t}(y)\right) \leq \varepsilon \text { for } t \geq 0 \\
\text { and } \lim _{t \rightarrow \infty} d\left(\varphi_{t}(x), \varphi_{t}(y)\right)=0
\end{array}
\end{array}\right\}, \\
& W_{\varepsilon}^{u}(x)=\left\{\begin{array}{ll}
y \in M & \begin{array}{l}
d\left(\varphi_{t}(x), \varphi_{t}(y)\right) \leq \varepsilon \text { for } t \leq 0 \\
\text { and } \lim _{t \rightarrow-\infty} d\left(\varphi_{t}(x), \varphi_{t}(y)\right)=0
\end{array}
\end{array}\right\} .
\end{aligned}
$$

These sets play the role of coordinates ([7], [10]). For each small $\varepsilon>0$, there is $\delta(\varepsilon)>0$ for which the following is true; whenever $x, y \in M$ satisfy $d(x, y) \leq \delta(\varepsilon)$ there is a unique $\mu=\mu(x, y) \in[-\varepsilon, \varepsilon]$ such that

$$
W_{\varepsilon}^{s}\left(\varphi_{\mu}(x)\right) \cap W_{\varepsilon}^{u}(y) \neq \phi,
$$

and this set consists of a single point, which is denoted by $\langle x, y\rangle$.

Let $D$ be a differentiable closed disk transverse to the flow $\varphi_{t}$ of dimension $\operatorname{dim}(M)-1$. If its diameter is sufficiently small, there is $\xi>0$ such that $(z, t) \rightarrow \varphi_{t}(z)$ gives a diffeomorphism of $D \times[-\xi, \xi]$ to $\varphi_{[-\hat{\xi}, \xi]}(D)$. For a set $T \subset D$ disjoint from the boundary $\partial D$ of $D$ and of small diameter compared with $d(T, \partial D)$, we can define

$$
\langle,\rangle_{D}: T \times T \rightarrow D
$$

by $\langle x, y\rangle_{D}=\operatorname{Pr}_{D}\langle x, y\rangle$, where $\operatorname{Pr}_{D}: \varphi_{[-\hat{\xi}, \hat{*}]}(D) \rightarrow D$ is the projection defined by $\operatorname{Pr}_{D}\left(\varphi_{t}(z)\right)=z$. We call $T$ a rectangle if $\langle x, y\rangle_{D} \in T$ for any $x, y \in T$. For a rectangle $T$ and $x \in T$ we set

$$
\begin{aligned}
& W^{s}(x, T)=\left\{\langle x, y\rangle_{D} \mid y \in T\right\}, \\
& W^{u}(x, T)=\left\{\langle y, x\rangle_{D} \mid y \in T\right\} .
\end{aligned}
$$

A proper family $\mathscr{T}$ of small size is said to be Markov if

1) each $T \in \mathscr{T}$ is a rectangle,

2) $W^{s}(x, T) \subset U(T, S)$ whenever $x \in U(T, S)$,

3) $W^{u}(x, T) \subset V(S, T)$ whenever $x \in V(S, T)$,

where

$$
\begin{aligned}
& U(T, S)=\mathrm{Cl}\left\{y \in T \cap \Gamma^{\prime}(\mathscr{T}) \mid H_{\mathscr{T}}(y) \in S\right\}, \\
& V(S, T)=\mathrm{Cl}\left\{y \in T \cap \Gamma^{\prime}(\mathscr{T}) \mid H_{\mathscr{T}}^{-1}(y) \in S\right\},
\end{aligned}
$$

and $\mathrm{Cl}(A)$ denotes the closure of a set $A$.

Given a proper family $\mathscr{T}$ we set 


$$
\mathscr{E}=\left\{(T, S) \in \mathscr{T} \times \mathscr{T} \begin{array}{l}
\text { there is } x \in T \cap \Gamma^{\prime}(\mathscr{T}) \\
\text { with } H_{\mathscr{F}}(x) \in S
\end{array}\right\}
$$

and define an oriented graph by $(\mathscr{T}, \mathscr{E})$ and a subshift of finite type $\Sigma(\mathscr{T}, \mathscr{E})$ by

$$
\left\{\left(T_{k}\right) \in \prod_{Z} \mathscr{T} \mid\left(T_{k}, T_{k+1}\right) \in \mathscr{E} \text { for any } k \in Z\right\} .
$$

It is known [5] that this subshift coincides with $Q\left(\Gamma^{\prime}(\mathscr{T})\right)$ if and only if $\mathscr{T}$ is Markov. In this case we can define a suspension $\Sigma\left(\mathscr{T}, \mathscr{E}, f_{\mathscr{T}}\right)$ as the set

$$
\left\{(X, s) \mid X \in \Sigma(\mathscr{T}, \mathscr{E}), 0 \leq s \leq f_{\mathscr{T}}(X)\right\}
$$

with $\left(X, f_{\mathscr{T}}(X)\right)$ and $(\sigma(X), 0)$ identified, where $\sigma: \Sigma(\mathscr{T}, \mathscr{E}) \rightarrow \Sigma(\mathscr{T}, \mathscr{E})$ is the shift operator given by $\sigma(X)_{k}=X_{k+1}$. On this space there is a vertical

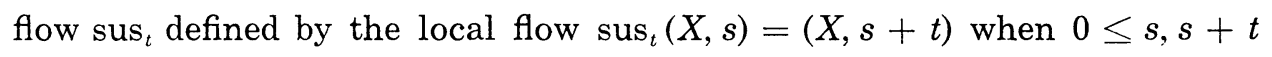
$\leq f_{\mathscr{T}}(X)$. Let $\rho: \Sigma\left(\mathscr{T}, \mathscr{E}, f_{\mathscr{T}}\right) \rightarrow M$ denote the surjective map given by $\rho(X, s)=\varphi_{s}(\pi(X))$. Then this map connects the suspension flow sus $s_{t}$ and the flow $\varphi_{t}$ in the following sense;

1) $\rho \circ \operatorname{sus}_{t}=\varphi_{t} \circ \rho$,

2) $\rho$ is a bounded-to-one map.

Now we state our result.

Theorem 1. Let $\varphi_{t}: M \rightarrow M$ be an Anosov flow. Suppose there is an involution $\theta: M \rightarrow M$ with $\varphi_{t} \circ \theta=\theta \circ \varphi_{-t}$. Then there exists a Markov family Il for $\varphi_{t}$ of arbitrarily small size such that

1) $\mathscr{M}$ admits an involution $\tau: \mathscr{M} \rightarrow \mathscr{M}$,

2) $\tau$ is a graph isomorphism of $(\mathscr{M}, \mathscr{E})$ to $\left(\mathscr{M}, \mathscr{E}^{*}\right)$, where $\mathscr{E} *=$ $\{(y, x) \mid(x, y) \in \mathscr{E}\}$,

3) the suspending function $f_{\|}$satisfies $f_{\mathscr{N}}=f_{\mathscr{N}} \circ \bar{\tau} \circ \sigma$, where $\bar{\tau}: \Sigma(\mathscr{M}, \mathscr{E}) \rightarrow$ $\Sigma(\mathscr{M}, \mathscr{E})$ is given by $\bar{\tau}(X)_{k}=\tau\left(X_{-k}\right)$,

4) if we define $\bar{\theta}: \Sigma\left(\mathscr{M}, \mathscr{E}, f_{\mathscr{M}}\right) \rightarrow \Sigma\left(\mathscr{M}, \mathscr{E}, f_{\mathscr{K}}\right)$ by $\bar{\theta}(X, s)=(\bar{\tau} \circ \sigma(X)$, $\left.f_{\mathscr{H}}(X)-s\right)$, then $\rho \circ \bar{\theta}=\theta \circ \rho$.

\section{§3. Proof}

Since the Anosov property does not depend on a metric, we may suppose $\theta$ is an isometry. Hence the stable and unstable sets satisfy $\theta\left(W_{\varepsilon}^{s}(x)\right)=W_{\varepsilon}^{u}(\theta x)$ and if $T$ is a rectangle, so is $\theta(T)$.

Let $\alpha>0$ be a sufficiently small number compared with $\varepsilon, \delta(\varepsilon)$, the minimal period of $\varphi_{t}$ and so on. Cover $M$ by a finite number of flow boxes 
of small size along a dense orbit. From the assumption $\varphi_{t} \circ \theta=\theta \circ \varphi_{-t}$, there are differentiable closed disks $D_{-n}, \cdots, D_{-1}, D_{1}, \cdots, D_{n}$ transverse to $\varphi_{t}$ and closed rectangles $B_{i} \subset \operatorname{Int}\left(D_{i}\right)$ such that

1) $\theta\left(D_{i}\right)=D_{-i}$ and $\theta\left(B_{i}\right)=B_{-i}$,

2) $\operatorname{dim}\left(D_{i}\right)=\operatorname{dim}(M)-1$,

3) $\operatorname{diam}\left(D_{i}\right)<3 \alpha / 4$,

4) $M=\bigcup_{i=1}^{n} \varphi_{[-2 \alpha / 3,-\alpha / 3]}\left(\operatorname{Int}_{D_{i}}\left(B_{i}\right)\right)$,

5) for $i \neq j$, at least one of the sets $D_{i} \cap \varphi_{[0,2 \alpha]}\left(D_{j}\right)$ and $D_{\jmath} \cap \varphi_{[0,2 \alpha]}\left(D_{i}\right)$ is empty,

6) if $B_{i} \cap \varphi_{[-\alpha / 2, \alpha / 2]}\left(B_{j}\right) \neq \phi$ then $B_{i} \subset \varphi_{[-\alpha, \alpha]}\left(D_{j}\right)$.

For $i>0$ choose a closed rectangle $K_{i} \subset \operatorname{Int}_{D_{i}}\left(B_{i}\right)$ so that $M=$ $\bigcup_{i=1}^{n} \varphi_{[-3 a / 4,-\alpha / 4]}\left(K_{i}\right)$ and set $K_{-i}=\theta\left(K_{i}\right)$. Pick $\nu>0$ so that any set with diameter smaller than $4 \nu$ is contained in some $\varphi_{[-\alpha, \alpha]}\left(K_{i}\right), i>0$. One can find large $L>0$ depending on $\nu$ and a finite closed covering $\mathscr{V}_{i}$ of $K_{i}$, $i>0$, with

$$
\max _{|t| \leq L} \operatorname{diam}\left(\varphi_{t}(V)\right) \leq \nu \text { and } V=\operatorname{Int}_{D_{i}}(V)
$$

for every $V \in \mathscr{V}_{i}$. There are $a(V), b(V) \in[1, n]$ for each $V \in \mathscr{V}_{i}$ such that $B_{\nu}\left(\varphi_{-L}(V)\right) \subset \varphi_{[-\alpha, \alpha]}\left(K_{a\left(\nu^{\prime}\right)}\right)$ and $B_{\nu}\left(\varphi_{L}(V)\right) \subset \varphi_{[-\alpha, \alpha]}\left(K_{b(v)}\right)$ : Here $B_{\nu}\left(\varphi_{-L}(V)\right)=$ $\left\{x \in M \mid d\left(x, \varphi_{-L}(V)\right)<\nu\right\}$. For $i<0$, we define a covering of $K_{i}$ by $\mathscr{V}_{i}=$ $\theta\left(\mathscr{V}_{-i}\right)=\left\{\theta(V) \mid V \in \mathscr{V}_{-i}\right\}$ and set $a, b: \mathscr{V}_{i} \rightarrow[-n,-1]$ by $a(\theta(V))=-b(V)$ and $b(\theta(V))=-a(V)$. Consider maps

$$
\begin{aligned}
& g_{V}^{-}=P_{D_{a(V)}} \circ \varphi_{-L}: V \rightarrow K_{a(V)}, \\
& g_{V}^{+}=P_{D_{b(V)}} \circ \varphi_{L}: V \rightarrow K_{b(V)},
\end{aligned}
$$

where $P_{D_{i}}: \varphi_{[-a, a]}\left(D_{i}\right) \rightarrow D_{i}$ is the projection. We inductively define

$$
\begin{aligned}
& R_{i .0}=S_{i, 0}=K_{\imath} \text {, }
\end{aligned}
$$

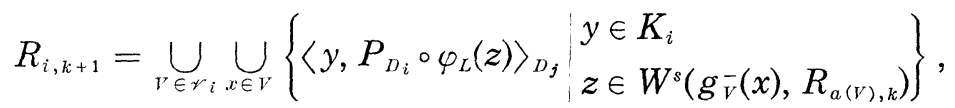

$$
\begin{aligned}
& S_{i, k+1}=\bigcup_{V \in r_{i} i} \bigcup_{x \in V}\left\{\begin{array}{l|l}
\left\langle P_{D_{i}} \circ \varphi_{-L}(z), y\right\rangle_{D_{i}} & \begin{array}{l}
y \in K_{i} \\
z \in W^{u}\left(g_{V}^{+}(x), S_{b(V), k}\right)
\end{array}
\end{array}\right\} .
\end{aligned}
$$

As $L$ is sufficiently large, it is not difficult to check that $R_{i, k}$ and $S_{i, k}$ are rectangles contained in $B_{i}$. Also we have $\theta\left(R_{i, k}\right)=S_{-i, k}$ because $\theta \circ g_{\vec{V}}^{+}=$ $g_{\theta(V)}^{\mp} \circ \theta$.

Now set rectangles $R_{\imath}=\bigcup_{k=0}^{\infty} R_{i, k}, S_{i}=\bigcup_{k=0}^{\infty} S_{i, k}$ and $C_{i}=\left\langle S_{i}, R_{i}\right\rangle_{D_{i}}=$ $\left\{\langle x, y\rangle_{D_{\imath}} \mid x \in S_{i}, y \in R_{i}\right\}$. Let $\mathscr{C}$ denotes the proper family $\left\{C_{i}\right\}_{i= \pm 1, \ldots, \pm n}$. By 
using the canonical bijection $H_{\mathscr{E}}: \Gamma(\mathscr{C}) \rightarrow \Gamma(\mathscr{C})$, we define $J^{+}(i)$ and $J^{-}(i)$ as the sets

$\left\{j \mid\right.$ there is $x \in \operatorname{Int}_{D_{i}}\left(C_{i}\right)$ with $\left.H_{\varphi}(x) \in \operatorname{Int}_{D_{j}}\left(C_{j}\right)\right\}$,

$\left\{j \mid\right.$ there is $x \in \operatorname{Int}_{D_{i}}\left(C_{i}\right)$ with $\left.H_{\mathscr{G}}^{-1}(x) \in \operatorname{Int}_{D_{j}}\left(C_{j}\right)\right\}$,

respectively. As $H_{\mathscr{C}} \circ \theta \circ H_{\mathscr{q}}=\theta$, we have $j \in J^{ \pm}(i)$ if and only if $-j \in$ $J^{\mp}(-i)$. From the choice of $\left\{D_{i}\right\}$, for each $j \in J(i)=J^{+}(i) \cup J^{-}(i), E_{i, j}=$ $C_{i} \cap P_{D_{i}}\left(C_{j}\right)$ is a rectangle with nonempty interior. Choose a point $z_{i, j} \in$ $\operatorname{Int}_{D_{i}}\left(E_{i, j}\right)$ for $j \in J^{+}(i)$ and put $z_{i, j}=\theta\left(z_{-i,-j}\right)$ for $j \in J^{-}(i)$. We part $C_{i}$ into four closed rectangles intersecting only in their boundaries;

$$
\begin{aligned}
& E_{\imath, j}^{1}=\mathrm{Cl}\left(\operatorname{Int}_{D_{i}}\left(E_{i, j}\right)\right), \\
& E_{i, j}^{2}=\mathrm{Cl}\left\{\begin{array}{l|l}
y \in \operatorname{Int}_{D_{i}}\left(C_{i}\right) \mid \begin{array}{l}
\left\langle z_{i, j}, y\right\rangle_{D_{i}} \in \operatorname{Int}_{D_{i}}\left(E_{i, j}\right) \\
\left\langle y, z_{i, j}\right\rangle_{D_{i}} \notin E_{i, j}
\end{array}
\end{array}\right\}, \\
& E_{i, j}^{3}=\mathrm{Cl}\left\{\begin{array}{l|l}
y \in \operatorname{Int}_{D_{i}}\left(C_{i}\right) \mid \begin{array}{l}
\left\langle z_{i, j}, y\right\rangle_{D_{i}} \notin E_{i, j} \\
\left\langle y, z_{i, j}\right\rangle_{D_{i}} \in \operatorname{Int}_{D_{i}}\left(E_{i, j}\right)
\end{array}
\end{array}\right\}, \\
& E_{i, j}^{4}=\mathrm{Cl}\left\{y \in \operatorname{Int}_{D_{i}}\left(C_{i}\right) \mid \begin{array}{l}
\left\langle z_{i, j}, y\right\rangle_{D_{i}} \notin E_{i, j} \\
\left\langle y, z_{i, j}\right\rangle_{D_{i}} \notin E_{i, j}
\end{array}\right\} .
\end{aligned}
$$

They satisfy $\theta\left(E_{i, j}^{1}\right)=E_{-i,-j}^{1}, \theta\left(E_{i, j}^{2}\right)=E_{-i,-j}^{3}, \theta\left(E_{i, j}^{3}\right)=E_{-i,-j}^{2}$ and $\theta\left(E_{i, j}^{4}\right)=$ $E_{-i,-j}^{4}$. We get a covering $\mathscr{E}_{i}$ of $C_{i}$ by a finite number of closed rectangles

$$
\mathscr{E}_{i}=\left\{\mathrm{Cl}\left(\bigcap_{j \in J(i)} \operatorname{Int}_{D_{i}}\left(E_{i, j}^{\ell(j)}\right)\right) \mid \ell: J(i) \rightarrow\{1,2,3,4\}\right\} .
$$

Put $U_{i}=\bigcup\left\{\operatorname{Int}_{D_{i}}(E) \mid E \in \mathscr{E}_{i}\right\}$ and set for a positive integer $N$

$$
\Gamma_{N}^{\prime}(\mathscr{E})=\left\{z \in \bigcup_{|i|=1}^{n} U_{i} \mid H_{\mathscr{E}}^{k}(z) \in \bigcup_{i} U_{i} \text { for }-N \leq k \leq N\right\} .
$$

Given $x, y \in \Gamma_{N}^{\prime}(\mathscr{E})$ we denote $x \sim y$ if for any $k \in[-N, N]$ there exists $E \in \cup_{i} \mathscr{E}_{i}$ with $H_{\mathscr{\varepsilon}}^{k}(x), H_{\mathscr{\varepsilon}}^{k}(y) \in E$. This is an equivalence relation. Since $\theta\left(U_{i}\right)=U_{-i}$ and $C_{i} \cap C_{-i}=\phi$, we have $x \neq \theta x$ and $x \sim y$ if and only if $\theta x \sim \theta y$. Let $G_{-m}, \cdots, G_{-1}, G_{1}, \cdots, G_{m}$ denote the equivalence classes, where we assign the indexes so that if $x \in G_{p}$ then $\theta x \in G_{-p}$. We pick very small numbers $0<u_{1}<\cdots<u_{m}$, put $u_{-p}=-u_{p}$ and set $\mathscr{M}_{N}=\left(\varphi_{u_{p}}\left(\bar{G}_{p}\right)\right)_{p= \pm 1, \ldots, \pm m}$.

Finally we should check that $\mathscr{M}_{N}$ is a Markov family for $\varphi_{t}$ if $N$ is sufficiently large compared with $L$. It is clear that $\mathscr{M}_{N}$ is a proper family of size $\alpha$. Suppose $x, y \in G_{p} \subset D_{i(p)}$ and consider $z=\langle x, y\rangle_{D_{i(p)}}$. Since at least one of the sets $D_{i} \cap \varphi_{[0,2 \alpha]}\left(D_{j}\right)$ and $D_{j} \cap \varphi_{[0,2 \alpha]}\left(D_{i}\right)$ is empty, we can inductively conclude that $H_{\mathscr{\varepsilon}}^{k}(x)$ and $H_{\mathscr{\varepsilon}}^{k}(y)$ are contained in the same $C_{i}$ 
and $H_{\mathscr{E}}^{k}(z)=\left\langle H_{\mathscr{f}}^{k}(x), H_{\mathscr{q}}^{k}(y)\right\rangle_{D_{i}}$ for each $k \in[-N, N]$. Therefore $z \in \bar{G}_{p}$ and $M_{p}=\varphi_{u_{p}}(\bar{G})$ is a rectangle.

Now suppose $x \in V\left(M_{q}, M_{p}\right) \cap \Gamma^{\prime}\left(\mathscr{M}_{n}\right)$. Choose arbitrary $y \in W^{u}\left(x, M_{p}\right) \cap$ $\varphi_{u_{p}}\left(G_{p}\right)$ and put $x^{\prime}=\varphi_{-u_{p}}(x), y^{\prime}=\varphi_{-u_{p}}(y) \in G_{p}$. We denote by $x_{1}, y_{1}$ the points $H_{\mathscr{q}}^{-N-1}\left(x^{\prime}\right), P_{D_{\ell}} \circ H_{\mathscr{q}}^{-N}\left(y^{\prime}\right) \in D_{\ell}$ respectively. As $N$ is sufficiently large, one can prove that $H_{\mathscr{q}}^{N+1}\left(y_{1}\right)=y^{\prime}$ and $H_{\mathscr{\varepsilon}}^{N}\left(x_{1}\right) \sim H_{\mathscr{c}}^{N}\left(y_{1}\right)$ just like the same way as in [5]. This implies that $H_{\mu_{N}}^{-1}(y) \in M_{q}$ and $y \in V\left(M_{q}, M_{p}\right)$. Hence $W^{u}\left(x, M_{p}\right) \subset V\left(M_{q}, M_{p}\right)$ whenever $x \in V\left(M_{q}, M_{p}\right)$. Similarly we can conclude $W^{s}\left(x, M_{p}\right) \subset U\left(M_{p}, M_{q}\right)$ if $x \in U\left(M_{p}, M_{q}\right)$. Therefore $\mathscr{M}=\mathscr{M}_{N}$ is a Markov family for $\varphi_{t}$ of size $\alpha$.

Define an involution $\tau: \mathscr{M} \rightarrow \mathscr{M}$ by $\tau\left(M_{p}\right)=M_{-p}$. Then it is clear that $\tau$ is a graph isomorphism of $(\mathscr{M}, \mathscr{E})$ to $(\mathscr{M}, \mathscr{E} *)$. Since $H_{\mathscr{M}} \circ \theta \circ H_{\mathscr{M}}=\theta$ and $t_{\mathscr{M}} \circ \theta \circ H_{\mathscr{k}}=t_{\mathscr{k}}$, we get that $f_{\mathscr{M}}=f_{\mathscr{M}} \circ \bar{\tau} \circ \sigma$ and that the induced map $\bar{\theta}$ : $\Sigma\left(\mathscr{M}, \mathscr{E}, f_{\mathscr{M}}\right) \rightarrow \Sigma\left(\mathscr{M}, \mathscr{E}, f_{\mathscr{M}}\right)$ satisfies $\theta \circ \rho=\rho \circ \bar{\theta}$ and $\bar{\theta} \circ \operatorname{sus}_{t}=\operatorname{sus}_{-t} \circ \bar{\theta}$.

\section{§4. Remarks}

We mention here about Markov partitions for Anosov diffeomorphisms. For the definition see for example [6]. If we slightly modify the definition of $\alpha$-pseudo-orbits, then along the line in [6] we have

Proposition 2. Let $f: M \rightarrow M$ be an Anosov diffeomorphism on a compact manifold $M$. Suppose there is an involution $\theta: M \rightarrow M$ with $f \circ \theta=$ $\theta \circ f^{-1}$. Then there exists a Markov partition $\mathscr{M}$ for $f$ of arbitrarily small size such that

1) $\theta$ induces an involutive graph isomorphism $\tau$ of the associated graph $(\mathscr{M}, \mathscr{E})$ to $(\mathscr{M}, \mathscr{E} *)$,

2) $\tau$ induces an involution $\bar{\theta}$ on the subshift of finite type $\Sigma(\mathscr{M}, \mathscr{E})$ with $\rho \circ \bar{\theta}=\theta \circ \rho$, where $\rho: \Sigma(\mathscr{M}, \mathscr{E}) \rightarrow M$ is the canonical map.

If a finite group $G$ acts isometrically on $M$ and satisfies $g \circ \varphi_{t}=\varphi_{ \pm t} \circ g$ ( $g \circ f=f^{ \pm} \circ g$ ) for each $g \in G$, then we can get a result of the same type. Also our proof is applicable to an Axiom $A$ flow restricted on a basic set which is invariant under the group action.

Addendum. After I wrote this paper M. Pollicott pointed me out Rees [11] has announced a part of Theorem 1. 


\section{REFERENCES}

[1] T. Adachi and T. Sunada, L-functions of pro-finite graphs and dynamical systems, To appear in J. Func. Anal.

[2] Homology of closed geodesics in a negatively curved manifold, To appear in J. Differential Geom.

[ 3 ] D. V. Anosov, Geodesic flows on closed Riemannian manifolds with negative curvature, Proc. Stekelov Inst. Math., 90, 1967.

[4] R. Bowen, Markov partitions for Axiom $A$ diffeomorphisms, Amer. J. Math., 92 (1970), 725-747.

[ 5 ] - - Symbolic dynamics for hyperbolic flows, Amer. J. Math., 95 (1973), 429-460.

[6] - Equilibrium state and the ergodic theory of Anosov diffeomorphisms, Lecture Note in Math. 470, Springer-Verlag, Berlin-New York, 1975.

[7] C. Pugh and M. Shub, $\Omega$-stability theorem for flows, Invent. math., 11 (1970), 150-158.

[8] M. Ratner, Markov partitions for Anosov flows on n-dimensional manifolds, Israel J. Math., 15 (1973), 92-114.

[ 9 ] C. Series, Symbolic dynamics for geodesic flows, Acta Math., 146 (1981), 103-128.

[10] S. Smale, Differentiable dynamical systems, Bull. Amer. Math. Soc., 73 (1967), 747-817.

[11] M. Rees, Checking ergodicity of some geodesic flows with infinite Gibbs measure, Ergod. Th. \& Dynam. Sys., 1 (1981), 107-133.

Depertment of Mathematics

Nagoya University

Chikusa-ku, Nagoya 464

Japan

Current Address:

Department of Mathematics

Faculty of General Education

Kumamoto University

Kurokami, Kumamoto 860

Japan 